\title{
INFORMALIDAD LABORAL Y HOMICIDIOS EN CALI, UNA CIUDAD DE ALTA VIOLENCIA*
}

Magaly Faride Herrera Giraldo ${ }^{a}$

Carlos Giovanni González Espitia

" DOI: https://doi.org/10.18601/01245996.v24n46.11. Recepción: 23-112020, modificación final: 27-10-2021, aceptación: 29-11-2021. Sugerencia de citación: Herrera G., M. F. y González E., C. G. (2022). Informalidad laboral y homicidios en Cali, una ciudad alta violencia. Revista de Economia Institucional, 24(46), 217-236.

a Doctora en Economía de los Negocios. Profesora Asistente del Departamento de Economía, Universidad Icesi, Cali, Colombia, [mfherrera@icesi. edu.co], [https://orcid.org/0000-0002-7377-1450]

b Doctor en Economía. Profesor Asociado del Departamento de Economía, Universidad Icesi, Cali, Colombia, [cggonzalez@icesi.edu.co], [https:// orcid.org/0000-0003-4807-7966]. 


\section{Informalidad laboral y homicidios en Cali, una ciudad de alta violencia}

Resumen En este artículo se estima el efecto de la informalidad laboral en los homicidios en Cali, mediante un análisis econométrico que usa variables instrumentales. Los resultados muestran un efecto positivo de la informalidad laboral (no estar afiliado al sistema de salud y no cotizar a pensión) sobre los homicidios: en promedio, un aumento de 1 punto porcentual en la tasa de informalidad laboral aumenta los homicidios en un 4,2\%, significativo al nivel del 1\%. Estos resultados son relevantes para el estudio de la economía del crimen en regiones en desarrollo.

Palabras clave: homicidios, informalidad laboral, variables instrumentales; JEL: K42, J46, C36

\section{Labor informality and homicides in Cali, a city oh high violence}

Abstract This paper estimates the effect of labor informality on homicides in Cali through an econometric analysis using instrumental variables. The results show a positive effect of labor informality (not being affiliated to the health system and not paying pension contributions) on homicides: on average, a one percentage point increase in the labor informality rate increases homicides by $4,2 \%$, significant at the $1 \%$ level. These results are relevant for the study of the economics of crime in developing regions.

Keywords: homicides, labor informality, instrumental variables; JEL: K42, J46, C36.

\section{Informalidade trabalhista e homicídios em Cali, uma cidade de alta violência}

Resumo Este artigo estima o efeito da informalidade do trabalho sobre os homicídios em Cali, por meio de uma análise econométrica que utiliza variáveis instrumentais. Os resultados mostram um efeito positivo da informalidade do trabalho (não estar vinculado ao sistema de saúde e não contribuir com pensão) sobre os homicídios: em média, um aumento de 1 ponto percentual na taxa de informalidade do trabalho aumenta os homicídios em 4,2\%, significativo no nível de 1\%. Esses resultados são relevantes para o estudo da economia do crime em regiões em desenvolvimento.

Palavras-chave: homicídios, informalidade do trabalho, variáveis instrumentais; JEL: $\mathrm{K} 42, \mathrm{~J} 46, \mathrm{C} 36$. 
- ste artículo analiza el efecto de la informalidad laboral en los - 1 homicidios en Cali, una de las principales ciudades de Colombia y una de las más violentas del mundo (Sánchez, Villaveces et al.,2011). Los homicidios han sido estudiados en la ciudad principalmente con un enfoque descriptivo y en los últimos años, la ciudad ha avanzado en su lucha contra el crimen a través de distintas estrategias como la creación del Observatorio de Seguridad por parte de la Alcaldía. Sin embargo, la tasa de homicidios sigue siendo una de las más altas comparada con el promedio nacional, así como con las de otras ciudades de Colombia y de América Latina.

Las estadísticas muestran que la ciudad es muy violenta, con una tasa bruta de mortalidad de 6,5 muertos por cada mil habitantes. De acuerdo con las cifras del Departamento Administrativo Nacional de Estadísticas (Dane), el 20\% de estas muertes son violentas entre las que se encuentran las relacionadas con homicidios, suicidios o accidentes que involucran vehículos motorizados. Además, el número de homicidios es sumamente alto, pues solo entre 2001 y 2015 hubo un total de 26.687 homicidios en la ciudad, cifra alarmante para cualquier ciudad del mundo. Esta cifra de homicidios es elevada incluso con respecto a regiones que han tenido conflictos armados; por ejemplo, en la guerra civil del Congo (1997-2002) fueron asesinadas $10 \mathrm{mil}$ personas solo en la ciudad de Brazzaville, Sendero Luminoso asesinó a 11.021 personas en Perú entre 1980 y 2000. En el Sahara Occidental el conflicto independentista dejó 15 mil muertos entre 1975 y 1991.

Cuadro 1

Diez ciudades más violentas del mundo, 2012

\begin{tabular}{lllccc}
\hline Lugar & Ciudad & País & Homicidios & Población & Homicidios $^{1}$ \\
\hline 1 & San Pedro Sula & Honduras & 1.411 & 753.990 & 187,1 \\
2 & Caracas & Venezuela & 4.364 & 3.247 .971 & 134,4 \\
3 & Acapulco & México & 940 & 833.294 & 112,8 \\
4 & Cali & Colombia & 1.930 & 2.319 .684 & 83,2 \\
5 & Maceió & Brasil & 795 & 996.733 & 79,8 \\
6 & Tegucigalpa & Honduras & 946 & 1.191 .111 & 79,4 \\
7 & Fortaleza & Brasil & 2.754 & 3.782 .684 & 72,8 \\
8 & Ciudad de Guatemala & Guatemala & 2.123 & 3.103 .685 & 68,4 \\
9 & Joao Pessoa & Brasil & 515 & 723.515 & 66,9 \\
10 & Barquisimeto & Venezuela & 804 & 1.242 .351 & 64,7 \\
\hline
\end{tabular}

${ }^{1}$ Número de homicidios por cada 100 mil habitantes.

Fuente: Most dangerous cities in the world, World Atlas, 2012.

E1 cuadro 1 muestra que Cali ocupó el cuarto puesto entre las ciudades más violentas del mundo en 2012, con 1.930 homicidios y una 
tasa de 83,2 homicidios por cada 100 mil habitantes. Una cifra que en ese año solo fue superada por Acapulco en México, Caracas en Venezuela y San Pedro Sula en Honduras.

El Código Penal Colombiano establece que comete homicidio "el que matare a otro" (Ley 599 de 2000, art. 103, modificado por el art. 14 de la ley 890 de 2004). Este análisis considera el homicidio común, en el cual el victimario tiene la intención de quitarle la vida a la víctima; no tiene en cuenta los suicidios ni homicidios en accidentes de tránsito ni aquellos en los que el victimario no tiene la intención de quitar la vida.

Ahora, relacionando los homicidios con el mercado de trabajo, se puede decir que dadas las condiciones no óptimas que hay en este mercado, muchas de las personas se enfrentan a la elección entre trabajar o cometer un delito (Gould, Weinberg y Mustard, 2002). De acuerdo con Grogger (1998), para los individuos que tienen condiciones laborales desfavorables es menos costoso cometer un delito que para quienes tienen condiciones laborales óptimas.

De acuerdo con la Organización Internacional del Trabajo (OIT) (2013), la economía informal se entiende como todas las actividades que, en la ley o en la práctica, no están cubiertas o están insuficientemente cubiertas por acuerdos formales. Desde este punto de vista, se revelan varios aspectos de la informalidad, uno es que los actores pueden ser informales porque carecen de protección social, no ejercen derechos en el trabajo y carecen de representación y voz en el lugar de trabajo. Por lo tanto, están excluidos de los beneficios que brinda el Estado, los mercados privados y los procesos políticos, y debido a esto, las personas asociadas a la economía informal se encuentran en gran medida desprotegidas, inseguras y vulnerables.

En Cali, la informalidad existe en una amplia gama de sectores y los actores de la economía informal a menudo están fuera del alcance de la protección social, la legislación laboral y las medidas de protección en el lugar de trabajo. Como señala la OIT (2013), los trabajadores informales a menudo no están organizados y, por esta y otras razones, no pueden articular sus derechos. Los lugares de trabajo pueden ser inseguros e insalubres con peligros graves $\mathrm{y}$, a veces, potencialmente mortales. Además, enfrentan largas jornadas laborales, la productividad y los ingresos suelen ser bajos. Es así como la ciudad presenta un mercado laboral segmentado con una parte formal donde los trabajadores tienen contratos laborales, acceso a la seguridad social y pensiones. Por otro lado, existe una parte informal en la cual las 
personas no tienen acceso a lo anterior, lo que las hace vulnerables para ser potenciales víctimas o victimarios.

De esta manera, en las economías en desarrollo, medidas como la tasa de desempleo en el mercado laboral formal no son buenos indicadores de las oportunidades laborales o la vulnerabilidad socioeconómica de las personas. En este caso, para la ciudad de Cali, la informalidad laboral es una mejor medida de las condiciones que afectan o condicionan los niveles de delincuencia.

El objetivo de este artículo es estimar el efecto de la informalidad laboral en los homicidios en la ciudad de Cali. Para ello, se realiza un análisis econométrico que utiliza un modelo en el cual la variable dependiente es el logaritmo natural del número de homicidios y se utiliza el método de variables instrumentales (VI) para identificar un efecto causal de la tasa de informalidad laboral sobre la variación de los homicidios. Con este fin, se utiliza los datos de la Encuesta de Empleo y de Calidad de Vida para Cali realizada por el Ministerio de Trabajo sobre las características laborales en 2012, los datos de los homicidios y otros delitos se obtienen de la Policía Nacional de Colombia y los datos de otras variables socioeconómicas y de control se obtienen de la Alcaldía de Cali consignados en los informes "Cali en Cifras".

Así, este artículo hace tres contribuciones. Primera, ayuda a entender la relación entre los homicidios y las variables del mercado laboral formal e informal en una ciudad violenta ubicada en un país en desarrollo. Segunda, innova el análisis incorporando variables de un mercado de trabajo dual o segmentado que captan el efecto de la informalidad laboral con varias definiciones de informalidad. Tercera, en las estimaciones se incluyen variables instrumentales.

Este artículo está compuesto por siete secciones, incluyendo esta introducción. En la segunda sección, se presenta un contexto sobre los homicidios y la informalidad en Cali. En la tercera sección, se presenta la metodología utilizada. En la cuarta, se presentan los datos y las variables. Posteriormente, en la quinta sección, se muestran los principales resultados y en la última, se presentan las conclusiones, limitaciones y recomendaciones de política.

\section{CONTEXTO}

En Colombia, los homicidios han mostrado una tendencia a la baja, pero esto no ha sucedido de igual forma en todas las ciudades. En la gráfica 1, se muestra la evolución de la tasa por cada 100 mil habitantes 
de las tres principales ciudades (Bogotá, Medellín y Cali) y del total nacional (Colombia), la tendencia nacional es a la baja, y las ciudades de Medellín y Bogotá han logrado alcanzar tasas muy cercanas al promedio nacional de 45 homicidios por cada 100 mil habitantes. Sin embargo, Cali parece un caso particular, ya que es la única de las grandes ciudades del país que no ha logrado converger al promedio nacional. Como se observa en la gráfica, la línea que representa los homicidios en la ciudad de Cali tiene una leve tendencia a la baja pero la brecha con respecto a la tasa nacional se mantiene y persiste en el tiempo.

Gráfica 1

Tasas de homicidios en Colombia, 2000-2015

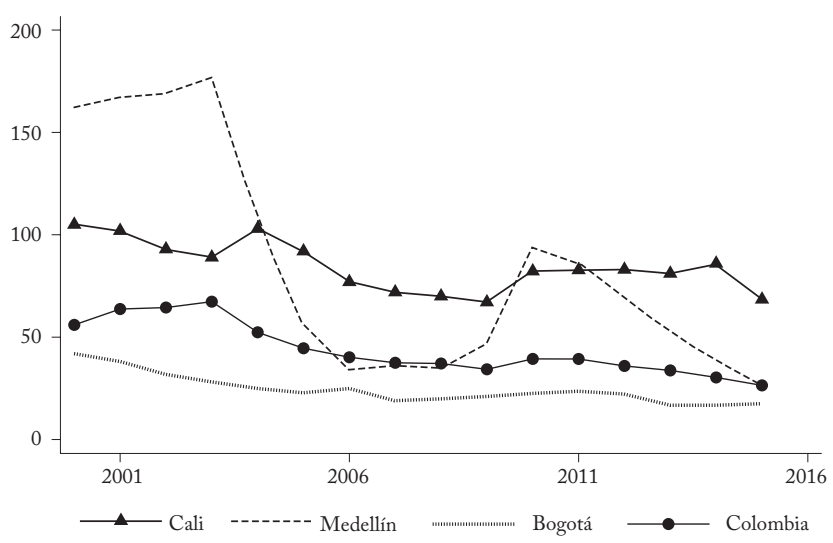

Fuente: Policía Nacional, elaboración propia.

Las cifras en la ciudad de Cali son alarmantes, a principios del siglo XXI, la tasa de homicidios era de 102 homicidios por cada 100 mil habitantes, durante los dos años siguientes se redujo un 13\%, pero en 2003 volvió a elevarse y alcanzó los 103 homicidios por cada 100 mil habitantes. A partir de dicho año, los homicidios presentaron una tendencia decreciente hasta 2008 y se redujeron un $25 \%$, 1legando a 67 homicidios por cada 100 mil habitantes, la tasa más baja de la década. Luego se presentó una tendencia creciente hasta 2013, cuando llegó a 86, en 2014 se redujo considerablemente, hasta 68,5; así, entre 2000 y 2015 los homicidios presentaron una reducción del $33 \%$. Sin embargo, como puede observarse, los homicidios en Cali se han mantenido por encima del promedio nacional.

En la gráfica 2 se presenta la evolución de la tasa de homicidios por cada 100 mil habitantes, la tasa de desempleo y una comparación de 
la relación entre estas dos variables. La tasa de desempleo, que refleja el comportamiento de las condiciones socioeconómicas, presentó una tendencia decreciente entre 2001 y 2008 hasta alcanzar el 12\%, luego se incrementó, para llegar al 15,4\% en 2011, y luego se redujo al 13,1\% en 2013; se observa entonces una fuerte correlación positiva entre los homicidios y la tasa de desempleo, tanto en su evolución como en su dispersión en el tiempo.

Gráfica 2

Tasa de homicidios por 100 mil habitantes y tasa de desempleo
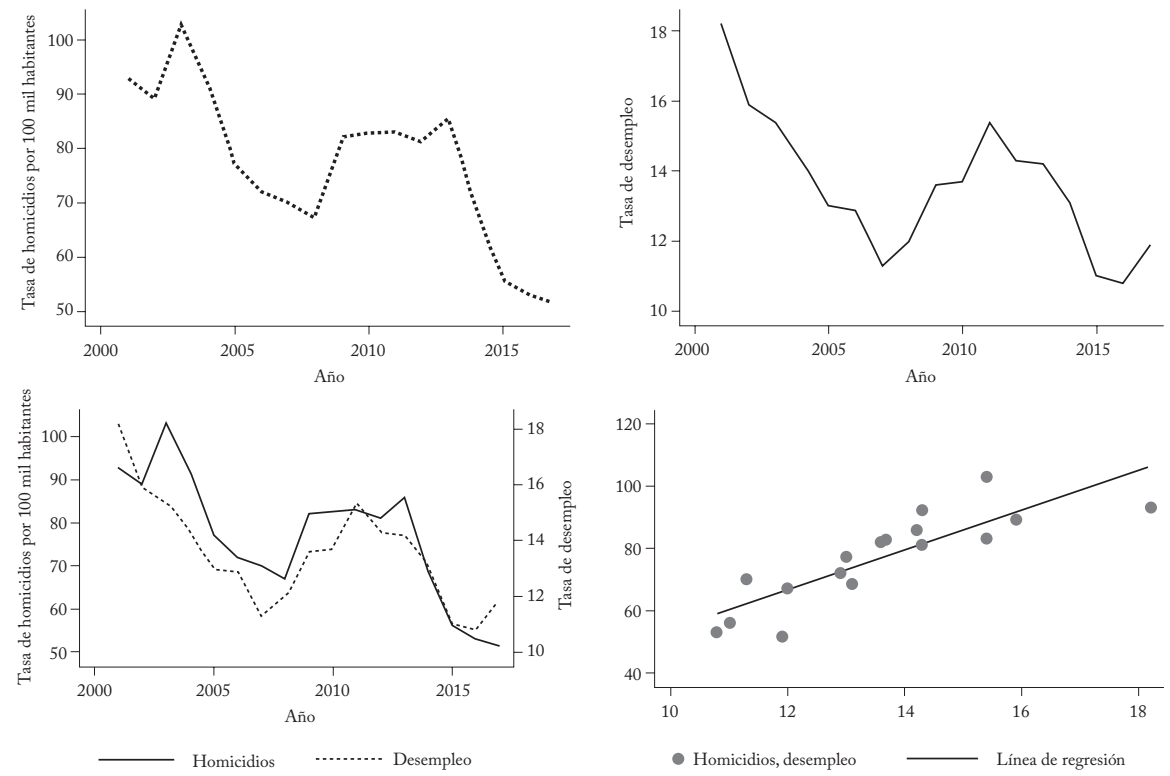

Fuente: Dane y Policía Nacional, elaboración propia.

Según González y Mora (2011), durante los primeros diez años del presente siglo el mercado laboral de Cali se encuentra segmentado en dos mercados, uno formal y el otro informal, llevando a la existencia de un mercado de trabajo dual. Es importante destacar que en el primero de estos grupos se encuentran los trabajadores que acceden a condiciones dignas de trabajo y en el segundo grupo se encuentran los trabajadores que tienen empleos de muy baja calidad, principalmente sin contrato laboral, sin afiliación al sistema de salud y que no cotizan a una pensión. De acuerdo con el Dane, la tasa de informalidad laboral en la ciudad era del 55,2\% en 2012, de modo que más de la mitad de la población laboral realizaba labores de muy baja calidad. En Colombia, Bernal (2009), Guataquí, García y Rodríguez 
(2011) y Sánchez (2013) muestran que utilizar las contribuciones a la seguridad social como una medida de informalidad es razonable. Otros artículos que analizan la informalidad laboral en Colombia son Mora $(2017,2021)$ y Mora y Muro (2017).

En este artículo se utilizan dos definiciones de informalidad, la primera es no tener contrato laboral, y se denomina informalidad relativa. La segunda definición es no tener afiliación al sistema de seguridad social en salud y no cotizar a una pensión, llamada informalidad aguda.

Varios autores han propuesto que los homicidios están correlacionados con el mercado laboral (Cantor y Land, 1985; Monahan y Klassen, 1982; Chalfin y Raphael, 2011; Dix, Soares y Ulyssea, 2018). La gráfica 2 muestra la existencia de una potencial relación positiva entre la tasa de desempleo y los homicidios en la ciudad de Cali, teniendo en cuenta esto, es posible afirmar que las políticas públicas orientadas a disminuir el desempleo indirectamente reducirían la tasa de homicidios en la ciudad.

De acuerdo con Cantor y Land (1985) esta relación puede ser positiva o negativa. Cuando esta relación es positiva se conoce en la literatura teórica del crimen como el efecto motivación en el cual las altas tasas de desempleo conllevan a que los individuos estén motivados a buscar otras posibilidades de supervivencia, entre ellas la opción de actividades criminales y por ende los homicidios. Esta situación puede entenderse como un aumento o disminución en el tamaño del mercado para el delincuente. Así cuando la economía va por buen camino y el desempleo es bajo, hay una mayor cantidad de personas que devengan un salario, por lo que las posibilidades de obtener un beneficio son mayores sin necesidad de realizar un gran esfuerzo. Por otro lado, cuando el desempleo aumenta, el hecho de matar se puede volver una opción para obtener ingresos.

Finalmente, puede haber una relación negativa entre la tasa de desempleo y los homicidios cuando las altas tasas de desempleo reducen el crimen y, por ello, los homicidios, porque las regiones con altas tasas de desempleo no son objetivo de la delincuencia. La oportunidad de cometer los delitos se presenta más en regiones donde la tasa de desempleo es más baja.

\section{METODOLOGÍA}

En esta sección se presenta la metodología para analizar la relación entre las variables del mercado laboral formal e informal y estimar 
el efecto causal que tiene la informalidad laboral en los homicidios en la ciudad de Cali. Para realizar el análisis econométrico se utiliza un modelo en el cual la variable dependiente es el logaritmo natural del número de homicidios de cada barrio de la ciudad y como variables independientes se consideran la tasa de desempleo y la tasa de informalidad de cada barrio. La unidad de análisis son los barrios en la ciudad. La ecuación (1) presenta una regresión simple de mínimos cuadrados ordinarios (MCO).

$Y_{i}=\beta_{0}+\beta_{1} X_{i}+\varepsilon_{i}$

donde $Y_{i}$ es el logaritmo del número de homicidios en un barrio $i, X_{i}$ es la tasa de desempleo del barrio $i$, la variable que se considera para analizar el efecto del mercado laboral formal y $\varepsilon_{i}$ es el término de error aleatorio. Con esta ecuación se pretende estimar la correlación estadística entre las dos variables. Sin embargo, hace falta incluir algunas variables de control para contrastar si en efecto existe una correlación entre el desempleo y los homicidios en la ciudad de Cali. La ecuación (2) incluye algunos controles asociados a otras características del mercado laboral, las dos variables de informalidad laboral: informalidad relativa e informalidad aguda.

$Y_{i}=\beta_{0}+\beta_{1} X_{i}+\Sigma \beta_{j} I_{j}+\varepsilon_{i}$

En esta ecuación $I_{j}$ representa las variables que capturan el efecto de la informalidad laboral. Adicionalmente, en la ecuación (3) se incluyen controles asociados a otros delitos, características de la población, características del barrio y de fuerza policial, $C_{j}$ para garantizar las mejores propiedades estadísticas del estimador que mide el efecto del mercado laboral sobre lo homicidios:

$Y_{i}=\beta_{0}+\beta_{1} X_{i}+\Sigma \beta_{j} I_{j}+\Sigma \alpha_{j} C_{j}+\varepsilon_{i}$

Es importante destacar que la estructura de los datos corresponde a un corte transversal de información de barrios de 2012. De modo que se estima el modelo por MCO con una unidad poblacional más agregada denominada "comuna" para garantizar la robustez del estimador y controlar el resultado debido al sesgo de agregación de datos (Durlauf, Navarro y Rivers, 2010).

Posteriormente, para estimar la relación causal entre la informalidad laboral y los homicidios se utilizó el método de variables instrumentales (VI) para obtener coeficientes con las mejores propiedades estadísticas posibles. La ecuación estructural del método es 
$Y_{i}=X_{i} \beta+\varepsilon_{i}$. Se asume que el supuesto de exogeneidad estricta no se encuentra $E[\varepsilon \mid X] \neq 0$, de esta manera la regresión por MCO se enfrenta a un problema de endogenidad. Para resolver el problema, el modelo se estima en dos etapas. La primera etapa $X_{i}=Z_{i}^{\prime} \pi+V_{i}$ aísla la parte de $X$ correlacionada con $\varepsilon_{i}$ usando variables instrumentales $Z_{i}$, para que los instrumentos sean válidos se debe cumplir: (i) relevancia cov( $z$, $X) \neq 0$ y (ii) exogeneidad $\operatorname{cov}(z, \varepsilon)=0$. Adicionalmente, de acuerdo con Andrews, Stock y Sun (2018) los instrumentos podrían ser débiles, lo cual significa que se debe probar que tan buenos son los instrumentos. Finalmente, la segunda etapa $Y_{i}=Z_{i}^{\prime} \delta+U_{i}$ es estimada incorporando la predicción de la variable endógena para la cual la correlación con el término de error aleatorio se aísla y se tiene en cuenta que $\delta=\pi \beta$ y $\varepsilon=U-\beta V$.

\section{DATOS Y VARIABLES}

En esta sección se describen los datos y variables utilizados para la estimación econométrica descrita en la metodología. La fuente para los datos del mercado laboral fue la Encuesta de Empleo y de Calidad de vida para Cali realizada por el Ministerio de Trabajo sobre las características laborales en 2012. Esta encuesta es única para la ciudad de Cali, ya que permite extrapolar los resultados a unidades de análisis más pequeñas que la ciudad, como los barrios y las comunas. La encuesta se realizó para un total de 8.600 hogares (30.458 personas encuestadas). Este tamaño de la muestra y su diseño garantizan un error de muestreo esperado no superior al 1,2\% para el total de Cali y no superior al $7 \%$ para los resultados en cada una de las comunas. Además, la encuesta permite analizar características demográficas como las condiciones de salud, la vivienda y la migración e identificar las particularidades de la dinámica laboral. La unidad de observación de esta encuesta es el hogar.

Cuadro 2

Estadísticas descriptivas de algunas variables del mercado laboral en Cali

\begin{tabular}{lcccccc}
\hline & $\begin{array}{c}\text { Informalidad } \\
\text { relativa }\end{array}$ & $\begin{array}{c}\text { Informalidad } \\
\text { aguda }\end{array}$ & $\begin{array}{c}\text { Tasa de } \\
\text { desempleo }\end{array}$ & $\begin{array}{c}\text { Tasa de } \\
\text { ocupación }\end{array}$ & $\begin{array}{c}\text { Estrato } \\
\text { moda }\end{array}$ & $\begin{array}{c}\text { Edad } \\
\text { promedio }\end{array}$ \\
\hline Media & $17,7 \%$ & $45,8 \%$ & $14,0 \%$ & $42,6 \%$ & 2,8 & 36 \\
Desv. est. & $16,2 \%$ & $19,8 \%$ & 10,90 & $10,1 \%$ & 1,2 & 6 \\
Observaciones & 298 & 298 & 298 & 298 & 298 & 298 \\
\hline
\end{tabular}

Fuente: Encuesta de Empleo y Calidad de Vida Cali, 2012, Ministerio del Trabajo, elaboración propia.

En el cuadro 2 se presentan algunas estadísticas descriptivas de las variables del mercado laboral y otras como la moda del estrato so- 
cioeconómico y la edad promedio de los habitantes del barrio. La variable del mercado laboral considerada como predictor del crimen es la tasa de desempleo (Cantor y Land, 1985), que en este caso tiene una media del 14\% por barrio y una desviación estándar del 10,9\%. Asimismo, la informalidad relativa (no tener contrato laboral) tiene un promedio del 17,7\% y una desviación estándar del 16,2\%, mientras que la informalidad aguda (definida como no tener afiliación al sistema de salud ni cotizar a pensión) tiene un promedio del 45,8\% y una desviación estándar del 19,8\%. Otras variables como el estrato moda tienen una media de 2,8 y una desviación estándar de 1,2; parece ser, entonces, que el estrato socioeconómico que más se repite es el estrato 3. La edad promedio de los habitantes de los barrios es de 36 años, con una desviación estándar de 6 años.

Los datos de homicidios y otros delitos se tomaron de las estadísticas de la Policía Nacional y la información sobre características del barrio o de la comuna se obtuvo de la Alcaldía de Cali. En el cuadro 3 se presentan las estadísticas descriptivas de algunos de los delitos que ocurrieron en los barrios de Cali en 2012. En promedio, se presentaron 7 homicidios por barrio, con una desviación estándar de 8 , un mínimo de 1 y un máximo de 42 homicidios. Ese mismo año se reportaron 28 hurtos a personas con un máximo de 262. Los otros hurtos considerados a la propiedad (residencias, motocicletas, automotores y celulares) se presentaron como mínimo una vez en cada barrio y la frecuencia fue mayor para el hurto a personas, seguido por el hurto a celulares y motocicletas. El hurto que menos se reportó fue el de hurto a residencias. Estas cifras evidencian un alto grado de violencia y un comportamiento heterogéneo entre los diferentes delitos que se comenten en los barrios de la ciudad.

Cuadro 3

Estadísticas descriptivas de algunos delitos

\begin{tabular}{lcccccc}
\hline & & \multicolumn{5}{c}{ Hurtos } \\
\cline { 3 - 7 } & Homicidios & Personas & Residencias & Motocicletas & Automotores & Celulares \\
\hline Media & 7 & 28 & 4 & 7,93 & 6 & 8,14 \\
$\begin{array}{l}\text { Desviación } \\
\text { estándar }\end{array}$ & 8 & 30 & 3,8 & 8 & 7,6 & 8,19 \\
Mínimo & 1 & 1 & 1 & 1 & 1 & 1 \\
Máximo & 42 & 262 & 25 & 57 & 36 & 60 \\
\hline
\end{tabular}

Fuente: Policía Nacional, elaboración propia.

Como se trata de entender mejor la relación entre el mercado laboral y la variación de los homicidios por barrios, es necesario empezar el 
análisis con las correlaciones estadísticas entre dichas variables. En la gráfica 3 se presentan dos diagramas de dispersión que muestran la distribución de los homicidios con la tasa de desempleo y la informalidad laboral relativa. La gráfica 3a muestra que hay una dispersión grande de los datos con una correlación negativa entre los homicidios y la tasa de desempleo. En la gráfica 3b se muestra una dispersión y una correlación positiva entre los homicidios y la tasa de informalidad laboral relativa. En este caso, las dos variables del mercado laboral seleccionadas (desempleo e informalidad) muestran potenciales efectos contradictorios sobre la variación de los homicidios en los barrios de la ciudad.

Gráfica 3

Diagrama de dispersión homicidios con variables del mercado laboral
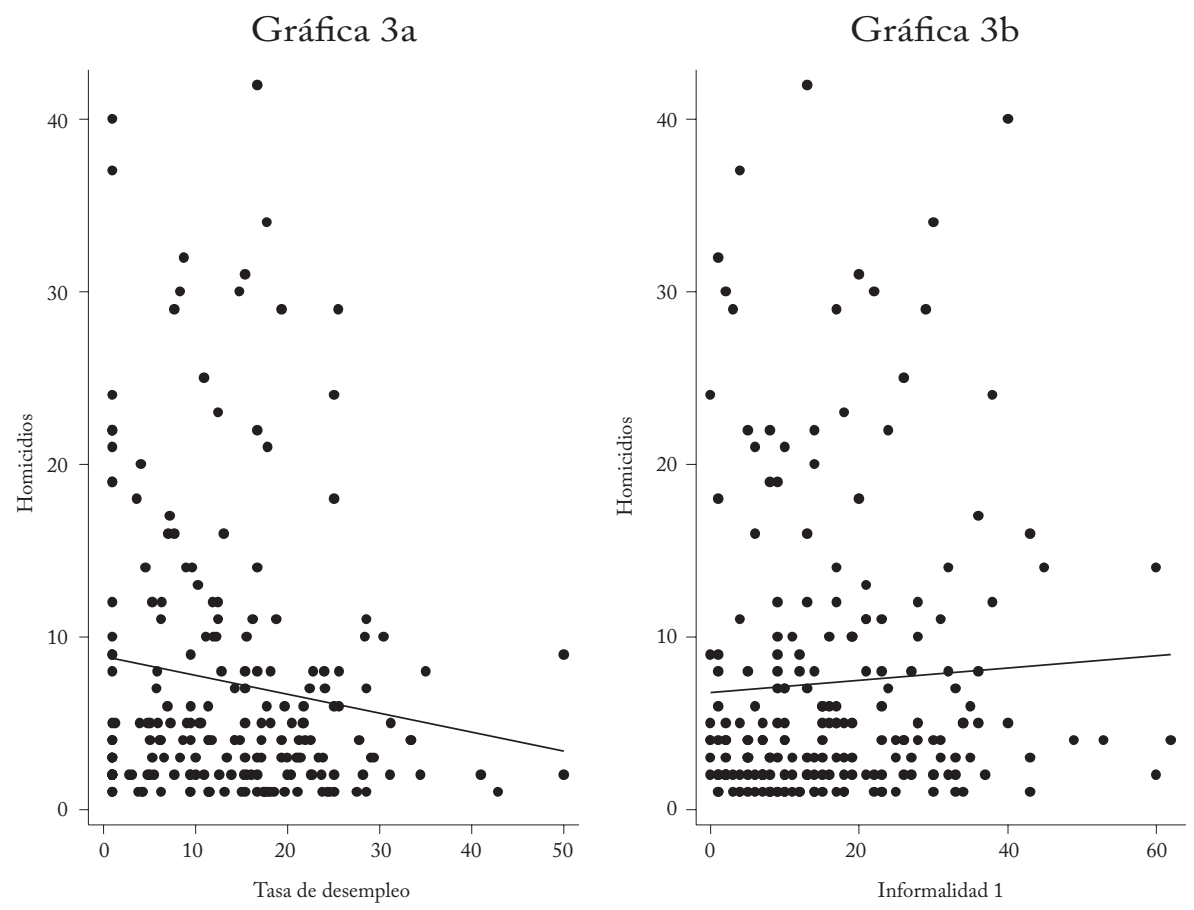

Fuente: Encuesta de Empleo y Calidad de Vida 2012, Cali. Ministerio del Trabajo, elaboración propia.

Para garantizar la mejor comprensión de la relación entre el mercado laboral y los homicidios en la ciudad es necesario realizar un análisis econométrico. A continuación, se presentan los resultados y la discusión de varios modelos estimados. 


\section{RESULTADOS}

Se hizo una regresión por MCO para estimar la relación entre las variables del mercado laboral formal e informal; para luego estimar el efecto causal que tiene la informalidad laboral en los homicidios en los barrios de la ciudad de Cali. El cuadro 4 contiene los resultados de la estimación del logaritmo de los homicidios según las variables del mercado laboral, la columna 1 muestra un efecto negativo y significativo de la tasa de desempleo sobre los homicidios. En las columnas 2, 3 y 4 se muestra la estimación que incluye como variables explicativas la tasa de desempleo y la tasa de informalidad laboral en sus dos definiciones. Los resultados de un efecto negativo de la tasa de desempleo sobre los homicidios se mantienen cuando las variables de informalidad se incluyen por separado y también cuando se consideran juntas. Esta distinción entre formalidad e informalidad es importante dado que la ciudad de Cali es una ciudad con un mercado laboral fuertemente segmentado.

Cuadro 4

Relación entre homicidios y variables del mercado laboral

\begin{tabular}{|c|c|c|c|c|}
\hline & $\mathrm{MCO}(1)$ & $\mathrm{MCO}(2)$ & $\mathrm{MCO}(3)$ & $\mathrm{MCO}(4)$ \\
\hline \multicolumn{5}{|c|}{ Variable dependiente: $\mathrm{Ln}$ (homicidios) } \\
\hline Constante & $0,7134^{* * * *}$ & $0,7127^{* * *}$ & $0,6577^{* * * *}$ & 0,6578 *** \\
\hline Tasa de desempleo & $-0,0049^{* * * *}$ & $-0,0048^{*}$ & $-0,0050 *$ & $-0,0050 *$ \\
\hline Informalidad relativa & & 0,0001 & & $-0,0001$ \\
\hline Informalidad aguda & & & 0,0034 & 0,0034 \\
\hline $\mathrm{R}^{2}$ & 0,014 & 0,014 & 0,024 & 0,025 \\
\hline $\mathrm{R}^{2}$ ajustado & 0,009 & 0,004 & 0,015 & 0,010 \\
\hline Observaciones & 211 & 211 & 211 & 211 \\
\hline
\end{tabular}

Nota: Las observaciones corresponden a los barrios de la ciudad. Todas las regresiones se hicieron con un error estándar robusto de tipo White.

$\mathrm{p}<0,01, " \mathrm{p}<0,05, " \mathrm{p}<0,1$.

Los resultados del modelo con variables del mercado laboral muestran que la tasa de desempleo (el mercado laboral formal) es buena para explicar los homicidios, pero la literatura reconoce la influencia de otras variables socioeconómicas y factores disuasivos que resultan relevantes para controlar el efecto del mercado laboral en los homicidios. En el cuadro 5 se muestra la estimación de tres modelos donde el estrato socioeconómico promedio de los hogares del barrio, la edad promedio de las personas del barrio, el área del barrio y otros delitos diferentes a los homicidios y otras variables son utilizadas como variables de control. 
De esta forma, la primera columna presenta el modelo con las variables antes mencionadas; además, incluye variables para controlar otro tipo de delitos como robos de celular y de motocicletas. E1 resultado a resaltar en esta estimación con variables de control es que la tasa de desempleo no es estadísticamente significativa y es la tasa aguda de informalidad la que es positiva y significativa para explicar la variación de los homicidios. Este resultado es la principal contribución del artículo, que muestra una fuerte evidencia de una relación positiva de la tasa aguda de informalidad laboral con los homicidios en una región en desarrollo y altamente violenta. Este resultado se puede explicar por la precariedad del mercado laboral en la ciudad con altos índices de informalidad y también porque hay un gran negocio entorno a matar gente en la ciudad, el sicariato o las conocidas en la ciudad como oficinas de sicarios.

Cuadro 5

Relación entre homicidios y variables del mercado laboral con variables de control

\begin{tabular}{|c|c|c|c|}
\hline & $\mathrm{MCO}(1)$ & $\mathrm{MCO}(2)$ & MCO (3) \\
\hline \multicolumn{4}{|c|}{ Variable dependiente: $\operatorname{Ln}$ (homicidios) } \\
\hline Constante & $0,9654^{* * * *}$ & $1,0149^{* * * *}$ & $1,0540^{* * * *}$ \\
\hline Tasa de desempleo & $-0,0023$ & $-0,0017$ & $-0,0011$ \\
\hline Informalidad relativa & $-0,0001$ & $-0,0001$ & $-0,0001$ \\
\hline Informalidad aguda & $0,0044^{* *}$ & $0,0088^{* *}$ & $0,0088^{* *}$ \\
\hline Estrato socioeconómico & $-0,2522^{* * *}$ & $-0,2429^{* * *}$ & $-0,2478^{* * *}$ \\
\hline Edad promedio & 0,0002 & $-0,0013$ & $-0,0022$ \\
\hline Área del barrio & $-0,0001^{* * * *}$ & $-0,0001^{* * * *}$ & $-0,0001^{* * *}$ \\
\hline Robo celulares & $0,0220^{* * * * *}$ & $0,0246^{* * * * *}$ & $0,0248^{* * * *}$ \\
\hline Robo Motocicletas & $0,0104^{* * * *}$ & $0,0089^{* * * *}$ & $0,0098^{* * * *}$ \\
\hline \multicolumn{4}{|l|}{ Variables de control } \\
\hline Otros crímenes & $\mathrm{Si}$ & $\mathrm{Si}$ & $\mathrm{Si}$ \\
\hline Características Población & - & $\mathrm{Si}$ & $\mathrm{Si}$ \\
\hline Características Barrio & - & $\mathrm{Si}$ & $\mathrm{Si}$ \\
\hline Características Policía & - & - & $\mathrm{Si}$ \\
\hline $\mathrm{R}^{2}$ & 0,4641 & 0,4712 & 0,4900 \\
\hline $\mathrm{R}^{2}$ ajustado & 0,4216 & 0,4234 & 0,4244 \\
\hline Observaciones & 211 & 211 & 211 \\
\hline
\end{tabular}

Nota: Las observaciones corresponden a los barrios de la ciudad. Todas las regresiones se hicieron con un error estándar robusto de tipo White. La palabra "Si" indica la inclusión de esas variables en la estimación. $\mathrm{p}<0,01,{ }^{+*} \mathrm{p}<0,05, \mathrm{p}<0,1$.

Se hicieron estimaciones que incluyen otras variables de control y los resultados se mantienen: un efecto positivo de la informalidad laboral aguda y un efecto nulo de la tasa de desempleo sobre la variación de 
los homicidios. Ahora bien, la columna 2 muestra la estimación con los controles de población y características del barrio (número de personas, número de mercados móviles, instituciones de salud, estaciones de bomberos y plazas de mercado) y la columna 3 muestra la estimación con los controles de persuasión policial (instituciones policiales, comisarías, subestaciones policiales, casas de justicia, comisarías familiares y centros de atención inmediata). Se encontró que el estrato tiene un efecto negativo y que el área del barrio, el robo de celulares y los mercados móviles tienen un efecto positivo en los homicidios.

Estos resultados permiten concluir que la parte formal del mercado laboral, específicamente la tasa de desempleo no está relacionada con los homicidios. Esto puede ocurrir porque existen condiciones que conllevan a que las personas sean más vulnerables en el mercado laboral, como la informalidad. Así se obtuvo que el efecto de la informalidad laboral aguda es significativo y positivo, por lo que hay un efecto del mercado laboral a los homicidios, pero es del mercado laboral informal, no del formal.

\section{ROBUSTEZ}

En este caso, los barrios se agregan en una observación mayor llamada comuna, que incluye los barrios formales e informales. Usamos la presencia policial como variable de control y encontramos que la relación entre tasa de homicidios e informalidad laboral aguda sigue siendo positiva y estadísticamente significativa, como muestra el cuadro 6. Si la estimación se controla por la población y el nivel de ingresos el resultado no cambia.

Cuadro 6

Relación entre homicidios y variables del mercado laboral, comunas

\begin{tabular}{|c|c|c|c|c|c|}
\hline & (1) & (2) & (3) & (4) & (5) \\
\hline \multicolumn{6}{|c|}{ Variable dependiente: Ln(homicidios) } \\
\hline Tasa de desempleo & 0,0062 & 0,0254 & 0,0358 & 0,0141 & 0,0141 \\
\hline Informalidad relativa & & $-0,0223$ & $-0,0208$ & $-0,0330$ & $-0,0310$ \\
\hline Informalidad aguda & & $0,0307^{*}$ & 0,0282 & $0,2213^{*+*}$ & $0,2300^{*}$ \\
\hline Presencia policial & & & 0,4735 & & $-0,0704$ \\
\hline Población & & & & $0,0007^{*}$ & $0,00073^{n+\infty}$ \\
\hline Ingreso per cápita & & & & & 0,00000273 \\
\hline
\end{tabular}

Nota: La regresión se hizo con un error estándar robusto de tipo White. $\mathrm{p}<0,01,{ }^{\cdots} \mathrm{p}<0,05,{ }^{\prime \prime} \mathrm{p}<0,1$.

La relación positiva entre homicidios e informalidad laboral aguda indica la vulnerabilidad de las personas para obtener una buena calidad 
de vida porque sus condiciones laborales no les permitan garantizar una fuente constante de ingresos y satisfacer sus necesidades. Como se mencionó antes, algunas personas enfrentan actividades marginadas y de supervivencia en la economía informal, esta situación crea un entorno propicio para convertirse en víctima o victimario de un homicidio. Así la informalidad laboral aguda explica mejor los homicidios que la tasa de desempleo, debido a que esta última no captura la complejidad de un mercado laboral precario como el de Cali.

Este resultado por comuna confirma los resultados encontrados por barrios, permitiendo comprender mejor la relación positiva y significativa entre los homicidios y la informalidad laboral aguda en la ciudad de Cali. Esto se debe a que en Cali una gran proporción de trabajadores se encuentra en la economía informal, por ejemplo, los subsidios por pérdida de empleo requieren que las personas tengan un contrato laboral formal. Por tanto, aquellas personas que se encuentran en una situación de informalidad laboral aguda, es decir, sin afiliación a salud y sin cotizar pensiones, tienden a ser más vulnerables y por lo tanto más propensas a cometer o ser victimas de homicidios.

\section{EVIDENCIA CAUSAL}

Aunque no existe literatura sobre el efecto de la informalidad laboral en los homicidios, en la regresión por el método de $\mathrm{MCO}$ puede haber un sesgo potencial debido a que es posible que la informalidad laboral esté correlacionada con el término de error aleatorio por varias razones: (a) la omisión de variables relevantes correlacionadas con las incluidas en el modelo, (b) posibles errores de medición de las variables independientes, (c) doble causalidad simultánea o (d) heterogeneidad no observada de los individuos. Esto causaría un problema de endogeneidad que llevaría a estimadores sesgados e inconsistentes, que impediría interpretar correctamente los resultados y sacar conclusiones.

Para atender el potencial problema se utilizó el método de variables instrumentales (VI). Este método aísla la parte de la informalidad laboral correlacionada con el término de error de tal manera que solo se estima la parte que realmente afecta los homicidios. El método utiliza variables instrumentales que deben satisfacer los supuestos de exogeneidad, relevancia e instrumentos fuertes (Andrews, Stock y Sun, 2018). Las variables instrumentales son: (a) el logaritmo del número de bancos y (b) el logaritmo del número de licencias de construcción. La estrategia de identificación detrás de las variables instrumentales es que la tasa de informalidad laboral aguda se correlaciona con el 
número de bancos y con el número de licencias de construcción, pero estos no afectan los homicidios. Las variables instrumentales siguen el supuesto de relevancia porque los barrios con mayor número de sucursales bancarias y licencias de construcción reducirán la tasa de informalidad laboral en esa zona, dado que por ley la contratación laboral se hace siguiendo las normas del mercado laboral vigentes.

Por otro lado, también se cumple el supuesto de exogeneidad, porque el número de bancos y licencias de construcción no son un determinante del comportamiento de los homicidios en la ciudad. Los instrumentos pueden estar asociados con otros delitos como hurto o atraco, pero no necesariamente relacionado con el homicidio común.

Cuadro 7

Efecto de la informalidad aguda en los homicidios

\begin{tabular}{lcccc}
\hline \multicolumn{1}{l}{ Variable dependiente: Ln(homicidios) } & & & \\
\hline & $\mathrm{MCO}(1)$ & $\mathrm{VI}(2)$ & $\mathrm{VI}(3)$ & $\mathrm{VI}(4)$ \\
\hline Informalidad aguda & $0,036^{* * *}$ & $0,055^{* * *}$ & $0,056^{* * * *}$ & $0,042^{* * * *}$ \\
Informalidad relativa & & & $-0,025$ & $-0,001$ \\
Variables instrumentales & & & & \\
\hline Ln(Licencias) & & $\mathrm{Si}$ & $\mathrm{Si}$ & $\mathrm{Si}$ \\
Ln(Bancos) & $\mathrm{Si}$ & $\mathrm{Si}$ & $\mathrm{Si}$ \\
Variables de control & & & $\mathrm{Si}$ \\
\hline Estadístico F & 7,36 & 5,77 & 7,20 & 154,53 \\
$\mathrm{R}^{2}$ ajustado & 0,2325 & 0,3332 & 0,4326 & 0,7996 \\
$1^{\text {a }}$ etapa estadístico F & & 5,56 & 9,58 & 16,20 \\
Prueba Sargan sobreindentificación & & 0,0128 & 0,0083 & 0,1080 \\
\hline
\end{tabular}

Kleibergen-Papp rk (Prueba F) 12,46

Prueba Anderson-Rubin

Nota: Las observaciones corresponden a los barrios de la ciudad.

$\mathrm{p}<0,01, " \mathrm{p}<0,05, " \mathrm{p}<0,1$.

E1 cuadro 7 muestra las estimaciones del efecto causal de la tasa de informalidad laboral aguda sobre la variación de los homicidios en los barrios de la ciudad de Cali. En la primera etapa, la estimación muestra que los instrumentos son relevantes porque tienen un $\mathrm{R}$ cuadrado alto (63\%), un valor $\mathrm{F}$ estadísticamente significativo con un nivel de confianza del $99 \%$ y sus coeficientes fueron estadísticamente significativos individualmente. La estimación en la segunda etapa (reportada en las columnas 2, 3 y 4) indica que cuando se utiliza los instrumentos de las tasas de informalidad en Cali, el logaritmo del número de bancos (Ln (Bancos)) y el logaritmo del número de licencias de construcción (Ln (Licencias)) y se controla por otras variables, se obtiene un efecto positivo. En promedio, un aumento de 
1 punto porcentual en la tasa de informalidad laboral aguda aumenta los homicidios en un $4,2 \%$, este resultado es significativo con un nivel de confianza del 99\%.

\section{CONCLUSIONES}

En una región en desarrollo que se caracteriza por su alta violencia, parece ser que el mercado laboral formal no es un buen determinante de los homicidios. Esto se debe principalmente a que existe una gran proporción de la población que se encuentra en un estado con mayor vulnerabilidad que es la informalidad laboral, una situación que resultaría más grave que estar desempleado en el mercado laboral formal, dado que una persona en la informalidad puede no tener contrato laboral (informalidad relativa) o no estar afiliado al sistema de salud y cotizar a pensiones (informalidad aguda).

Este artículo ayuda a entender la relación entre homicidios y variables del mercado laboral formal e informal, en una ciudad de alta violenta de un país en desarrollo. Se analiza la relación entre mercado laboral y homicidios incorporando variables de un mercado de trabajo dual o segmentado que captan el efecto de la informalidad laboral con varias definiciones de informalidad. Los barrios de Cali son la unidad de análisis de estas estimaciones. Como ejercicio de robustez se hacen estimaciones para las comunas, con los mismos resultados.

Por último, se estima el efecto causal de la informalidad laboral sobre los homicidios utilizando el método de variables instrumentales encontrando como resultado, un efecto positivo y estadísticamente significativo de la informalidad laboral aguda (no tener afiliación a salud y plan de pensiones) sobre los homicidios, así como un efecto nulo de la tasa de desempleo. En promedio, un aumento de 1 punto porcentual en la tasa de informalidad laboral aguda aumenta los homicidios en un $4,2 \%$, este resultado es significativo con un nivel de confianza del 99\%. De esta manera, cuando aumenta la tasa de informalidad aguda o se empeoran las condiciones del mercado laboral aumentan los homicidios en la ciudad de Cali, una región en desarrollo y altamente violenta.

\section{REFERENCIAS BIBLIOGRÁFICAS}

Alcaldía de Cali. (2012a). Observatorio de seguridad: Delitos contra la vida. Secretaría de Seguridad y Justicia, [https://www.cali.gov.co/ observatorioseguridad]. 
Alcaldía de Cali. (2012b). Cali en cifras, [https://planeacion.cali.gov.co/ caliencifras/Documentos\%20pdf/Caliencifras2012.pdf].

Andrews, I., Stock, J. y Sun, L. (2018). Weak instruments in IV regression: Theory and practice, working paper no publicado.

Bernal S, R. (2009). The informal labor market in Colombia: identification and characterization. Desarrollo y Sociedad, 63,145-208.

Cantor, D. y Land, K. C. (1985). Unemployment and crime rates in the post-World War II United States: A theoretical and empirical analysis. American Sociological Review, 50(3), 317-332.

Chalfin, A. y Raphael, S. (2011). Work and crime. En M. Tonry (ed.), The Oxford Handbook of Crime and Criminal Justice (pp. 444-476). Oxford: Oxford University Press.

Durlauf, S. N., Navarro, S. y Rivers, D. A. (2010). Understanding aggregate crime regressions. Journal of Econometrics, 158(2), 306-317.

Dix-C., R., Soares, R. R. y Ulyssea, G. (2018). Economic shocks and crime: Evidence from the Brazilian trade liberalization. American Economic Journal, 10(4), 158-195.

González, C. G. y Mora, J. J. (2011). Políticas activas de empleo para Cali-Colombia. Estudios Gerenciales, 27(118), 13-41.

Gould, E. D., Weinberg, B. A. y Mustard, D. B. (2002). Crime rates and local labor market opportunities in the United States: 1979-1997. Review of Economics and Statistics, 84(1), 45-61.

Grogger, J. (1998). Market wages and youth crime. Journal of Labor Economics, 16(4), 756-791.

Guataquí, J. C., García-S., A. F. y Rodríguez-A., M. (2011). E1 perfil de la informalidad laboral en Colombia. Universidad del Rosario: Serie Documentos de Trabajo No. 95.

Ministerio de Trabajo. (2012). Encuesta de empleo y calidad de vida de Cali 2012, [http://filco.mintrabajo.gov.co/FILCO/faces/datosAbiertos. jsf;jsessionid=oWHWbEKwKjpnaHFdAzbnP7AS8tsMcPCuREPCJW dQKwbizb9KPsVt!1160198892].

Monahan, J. y Klassen, D. (1982). Situational approaches to understanding and predicting individual violent behavior. En M. E. Wolfgang y N. A. Winter (eds.), Criminal violence, (pp. 292-319). Thousand Oaks, CA: Sage Publications.

Mora J. J. (2021). Análisis del desempleo y la ocupación después de una política estricta de confinamiento por COVID-19 en Cali. Lecturas de Economía, 94, 165-193.

Mora, J. J. (2017). La informalidad laboral colombiana en los últimos años: análisis y perspectivas de política pública. Revista de Métodos Cuantitativos para la Economía y la Empresa, 24(1), 89-128.

Mora, J. J. y Muro, J. (2017). Dynamic effects of the minimum wage on informality in Colombia, LABOUR, 31(1), 59-72.

OIT. (2013). Transición a la formalidad en América Latina y el Caribe: situación y tendencias. Panorama laboral 2013. Lima: OIT, Oficina Regional para América Latina y el Caribe.

Sánchez T., R. M. (2013). Enfoques, conceptos y metodologías de medición de la informalidad laboral en Colombia. Lecturas de Economía, 79, 9-43. 
Sánchez, Á. I., Villaveces et al. (2011). Policies for alcohol restriction and their association with interpersonal violence: a time-series analysis of homicides in Cali, Colombia. International Journal of Epidemiology, 40(4), 1037-1046. 